\section{Toothbrushes brushing off the dangers of alcohol}

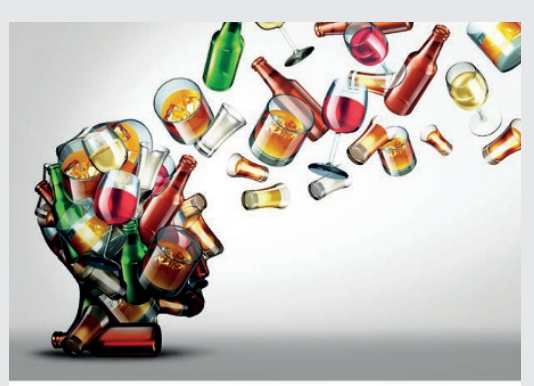

Alcohol is bad news for oral and general good health. Patients might not know just how much it can damage their teeth so it is the job of dental practitioners to educate them, plus give them a practical

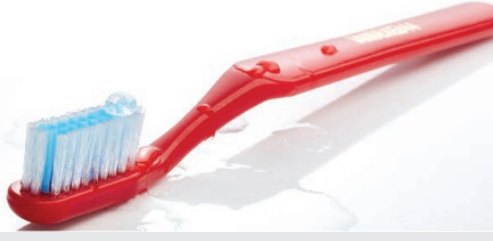
prevention plan.

As well as limiting consumption and regular visits to the dentist, every patient needs effective tools and

adjuncts. TANDEX produces a high-quality range of toothbrushes, including interdental and interspace brushes, for gentle yet effective cleaning of enamel weakened by the acid in alcoholic drinks.

Also available is PREVENT GEL, which is non-abrasive, can actively strengthen the enamel and contains an effective combination of fluoride and chlorhexidine. The handy tube is adapted for use over a three-week period, with the supervision of a dental practitioner.

For optimal protection, patients can supplement their routine with PREVENT mouthwash, which is available in two varieties. PREVENT WASH is double active, with fluoride and chlorhexidine and PREVENT FRESH is triple active, containing zinc too for effective protection against bad breath.

TANDEX is the first line of defence against oral health compromised by alcohol consumption.

For more information on Tandex's products, visit www. tandex.dk or visit the Facebook page: www.facebook.com/pages/ Tandex-UK/234855250044190?fref=ts.

\section{Resolve the issues surrounding RPDs}

To improve the fit, function and aesthetics of removable partial dentures (RPDs), practitioners are being invited to discover Ultaire AKP.

Ultaire AKP is a high performance polymer customdesigned by Solvay Dental 360 specifically for the fabrication of removable partial denture frames.

This new generation material offers patients a lightweight, comfortable, metal-free and taste-free option with strong, stable retention and unique 'snap in fit' clasp designs for superior aesthetic results.

More information is available at www.solvaydental360.com.

\section{D technology within your grasp}

If you thought powerful imaging technology was out of reach, think again. The CS 8100 3D from Carestream Dental has a broad range of applications and is a high-value investment for any practice that aims for diagnostics and treatment planning excellence.

Suitable for daily use, the CS 8100 3D system offers:

- Precision from every angle

- Versatility - it can be used for implant planning, endodontics, orthodontics, oral surgeries and more

- Safety with low radiation dosage

- Practicality as the compact unit can fit into small spaces and has minimal training requirements

- Convenience and comfort for all types of patient as images are acquired quickly.

The CS $81003 \mathrm{D}$ is just one product from Carestream Dental, which says it has an unrivalled reputation for innovation, cutting-edge technology and superior customer care.

More information is available by contacting Carestream Dental on 08001699692 or visiting www.carestreamdental.co.uk.

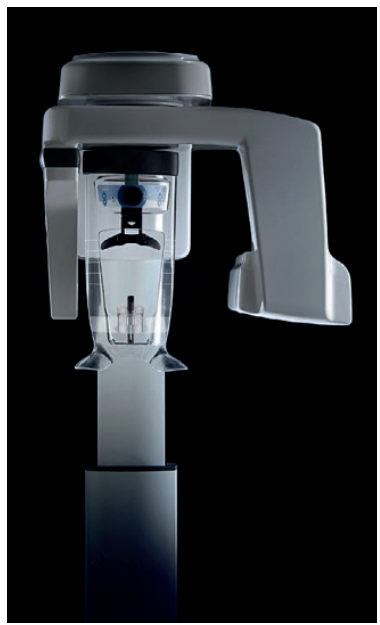

\section{Your defence against drug resistant infections}

Did you know that some health threatening pathogens such as klebsiella pneumoniae are quickly forming drug resistant strains?

These bacteria are responsible for a number of debilitating illnesses such as pneumonia and can be spread through simple human-to-human contact.

Defend against these threats with the Steri-7 Xtra range of products from Initial Medical.

Usable as both biocidal cleaners and high-level disinfectants, these products effectively eradicate bacteria, spores, fungi, yeasts and viruses, including those responsible for drug resistant strains of infections.

For more information visit www.initial.co.uk/medical or call 08708504045 .
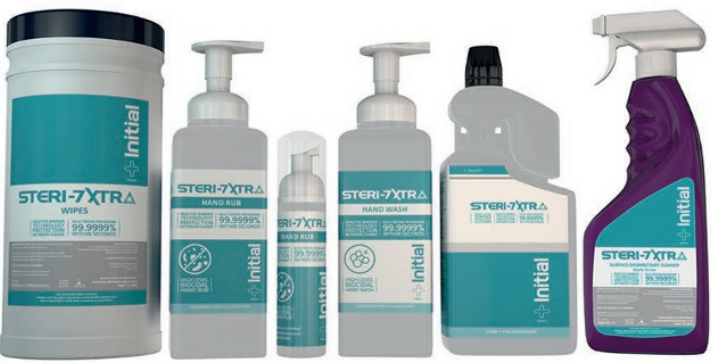